\title{
MEASUREMENT OF THE DECAY RATE AND FORM FACTOR PARAMETER $\alpha_{\mathbf{K}^{*}}$ IN THE DECAY $\mathrm{K}_{\mathbf{L}} \rightarrow \mathrm{e}^{+} \mathrm{e}^{-} \gamma$
}

\author{
NA48 Collaboration
}

\begin{abstract}
The decay rate of the neutral $\mathrm{K}$ meson $\mathrm{K}_{\mathrm{L}} \rightarrow \mathrm{e}^{+} \mathrm{e}^{-} \gamma$ has been measured with the NA48 detector at the CERN SPS. A total of 6864 events has been observed with an estimated background of 10 events. The branching ratio is $\Gamma\left(\mathrm{K}_{\mathrm{L}} \rightarrow \mathrm{e}^{+} \mathrm{e}^{-} \gamma\right) / \Gamma\left(\mathrm{K}_{\mathrm{L}} \rightarrow\right.$ all $)=\left(1.06 \pm 0.02_{\text {stat. }} \pm 0.02_{\text {sys. }} \pm 0.04_{\text {calc. }}\right) \times 10^{-5} . \quad$ The parameter $\alpha_{\mathrm{K}^{*}}$ describing the relative strength of the two contributing amplitudes to this decay through intermediate pseudoscalar or vector mesons, was measured to be $\alpha_{K^{*}}=-0.36 \pm 0.06_{\text {stat. }} \pm 0.02_{\text {sys. }}$.
\end{abstract}




\section{MEASUREMENT OF THE DECAY RATE AND FORM FACTOR PARAMETER $\alpha_{K^{*}}$ IN THE DECAY $K_{\mathbf{L}} \rightarrow \mathbf{e}^{+} \mathbf{e}^{-} \gamma$}

V. Fanti, A. Lai, L. Musa ${ }^{1}$, D. Marras, A. Nappi

Dipartimento di Fisica dell'Università e Sezione dell'INFN di Cagliari, I-09100 Cagliari, Italy.

B. Hay, R.W. Moore ${ }^{2}$, K.N. Moore ${ }^{2}$, D.J. Munday, M.D. Needham 5 , M.A. Parker, T.O. White, S.A. Wotton

Cavendish Laboratory, University of Cambridge, Cambridge CB3 OHE, U.K. ${ }^{3}$.

G. Barr, G. Bocquet, J. Bremer, A. Ceccucci, D. Cundy, N. Doble, W. Funk, L. Gatignon, A. Gianoli, A. Gonidec, G. Govi,

P. Grafström, W. Kubischta, A. Lacourt, S. Luitz, G. Kesseler, J.P. Matheys, A. Norton, S. Palestini,

B. Panzer-Steindel, D. Schinzel, H. Taureg, M. Velasco, O. Vossnack, H. Wahl, G. Wirrer

CERN, CH-1211 Geneva 23, Switzerland.

V. Kekelidze, A. Mestvirishvili, I. Potrebenikov, G. Tatishvili, A. Tkatchev, A. Zinchenko

Joint Institute for Nuclear Research, Dubna, Russian Federation.

O. Boyle, V.J. Martin, I.G. Knowles, H.L.C. Parsons

Department of Physics and Astronomy, University of Edinburgh, JCMB King's Buildings, Mayfield Road, Edinburgh, EH9 3JZ U.K. .

P. Dalpiaz, J. Duclos, P.L. Frabetti, M. Martini, F. Petrucci, M. Porcu, M. Savrié

Dipartimento di Fisica dell'Università e Sezione dell'INFN di Ferrara, I-44100 Ferrara, Italy.

A. Bizzeti ${ }^{14}$, M. Calvetti, G. Collazuol, G. Graziani, E. Iacopini, M. Lenti, A Michetti

Dipartimento di Fisica dell'Università e Sezione dell'INFN di Firenze, I-50125 Firenze, Italy.

H.G. Becker, H. Blümer ${ }^{1}$, P. Buchholz ${ }^{6}$, D. Coward ${ }^{7}$, C. Ebersberger, H. Fox, A. Kalter, K. Kleinknecht, U. Koch, L. Köpke, B. Renk, J. Scheidt, J. Schmidt, V. Schönharting, Y. Schué, R. Wilhelm, A. Winhart, M. Wittgen

Instut für Physik, Universität Mainz, D-55099 Mainz, Germany ${ }^{8}$.

J.C. Chollet, S. Crépé, L. Iconomidou-Fayard, L. Fayard, J. Ocariz ${ }^{9}$, G. Unal, D. Vattolo, I. Wingerter

Laboratoire de l'Accélérateur Linéaire, IN2P3-CNRS, Université de Paris-Sud, F-91406 Orsay, France ${ }^{10}$.

G. Anzivino, P. Cenci, P. Lubrano, M. Pepe

Dipartimento di Fisica dell'Università e Sezione dell’INFN di Perugia, I-06100 Perugia, Italy.

B. Gorini ${ }^{1}$, P. Calafiura, R. Carosi, C. Cerri, M. Cirilli, F. Costantini, R. Fantechi, S. Giudici, I. Mannelli, V. Marzulli,

G. Pierazzini, M. Sozzi

Dipartimento di Fisica dell'Università, Scuola Normale Superiore e Sezione dell'INFN di Pisa, I-56100 Pisa, Italy.

J.B. Cheze, J. Cogan, M. De Beer, P. Debu, A. Formica, R. Granier de Cassagnac, P. Hristov ${ }^{11}$, E. Mazzucato, B. Peyaud, S. Schanne, R. Turlay, B. Vallage

DSM/DAPNIA - CEA Saclay, F-91191 Gif-sur-Yvette, France.

I. Augustin, M Bender, M. Holder, M. Ziolkowski

Fachbereich Physik, Universität Siegen, D-57068 Siegen, Germany ${ }^{12}$.

R. Arcidiacono, C. Biino, F. Marchetto, E. Menichetti

Dipartimento di Fisica dell'Università e Sezione dell'INFN di Torino, I-10125 Torino, Italy.

J.Nassalski, E. Rondio, M. Szleper, W. Wislicki, S. Wronka

Soltan Institute for Nuclear Studies, Laboratory for High Energy Physics, PL-00-681 Warsaw, Poland ${ }^{13}$.

H. Dibon, G. Fischer, M. Jeitler, M. Markytan, I. Mikulec, G. Neuhofer, M. Pernicka, A. Taurok

Österreichische Akademie der Wissenschaften, Institut für Hochenergiephysik, A-1050 Wien, Austria ${ }^{15}$. 
${ }^{1}$ Present address: CERN, CH-1211 Geneva 23, Switzerland.

${ }^{2}$ Present address: Physics-Astronomy Building, Michigan State University, East Lansing, MI 48824 U.S.A..

${ }^{3}$ Funded by the U.K. Particle Physics and Astronomy Research Council.

${ }^{4}$ Permanent address: Dipartimento di Fisica dell'Università e Sezione dell'INFN di Torino, I-10125 Torino, Italy.

${ }^{5}$ Corresponding author: matthew.needham@cern.ch. Present address: NIKHEF, PO Box 41882, 1009 DB Amsterdam, The Netherlands.

${ }^{6}$ Present address: Institute für Physik, Universität Dortmund. D-44221 Dortmund, Germany.

${ }^{7}$ Present address: SLAC, Stanford, CA 94309, U.S.A..

${ }^{8}$ Funded by the German Federal Minister for Research and Technology (BMBF) under contract 7MZ18P(4)-TP2.

${ }^{9}$ Permanent address: Departamento de Física, Universidad de los Andes, Mérida 5101-A, Venezuela.

${ }^{10}$ Funded by Institut National de Physique des Particules et de Physique Nucléaire (IN2P3), France.

${ }^{11}$ Permanent address: Joint Institute for Nuclear Research, Dubna, Russian Federation.

${ }^{12}$ Funded by the German Federal Minister for Research and Technology (BMBF) under contract 056SI74.

${ }^{13}$ Funded by the Committee for Scientific Research (KBN), grant 2 P03B07615.

${ }^{14}$ Also Dipartimento di Fisica dell'Università di Modena, I-41100 Modena, Italy.

${ }^{15}$ Funded by the Bundesministerium f. Wissenschaft und Verkehr under the contract GZ 616.360/3-III/2a/98.

\begin{abstract}
The decay rate of the neutral $\mathrm{K}$ meson $\mathrm{K}_{\mathrm{L}} \rightarrow \mathrm{e}^{+} \mathrm{e}^{-} \gamma$ has been measured with the NA48 detector at the CERN SPS. A total of 6864 events has been observed with an estimated background of 10 events. The branching ratio is $\Gamma\left(\mathrm{K}_{\mathrm{L}} \rightarrow \mathrm{e}^{+} \mathrm{e}^{-} \gamma\right) / \Gamma\left(\mathrm{K}_{\mathrm{L}} \rightarrow\right.$ all $)=\left(1.06 \pm 0.02_{\text {stat. }} \pm 0.02_{\text {sys. }} \pm 0.04_{\text {calc. }}\right) \times 10^{-5}$. The parameter $\alpha_{\mathrm{K}^{*}}$ describing the relative strength of the two contributing amplitudes to this decay through intermediate pseudoscalar or vector mesons, was measured to be $\alpha_{K^{*}}=-0.36 \pm 0.06_{\text {stat. }} \pm 0.02_{\text {sys. }}$.
\end{abstract}




\section{Introduction}

The study of the decay of the long-lived neutral $\mathrm{K}$ meson into $\mathrm{K}_{\mathrm{L}} \rightarrow \ell^{+} \ell^{-} \gamma$ sheds light on the structure of the $\mathrm{K}_{\mathrm{L}} \rightarrow \gamma{ }^{*}$ vertex from which the virtual photon produces the lepton pair [1]. In phenomenological models of this structure, the possible rôles of vector meson dominance in the photon propagator [2], penguin and pole diagrams [3], and higher order terms in chiral perturbation theory [4] have been explored. Bergström, Massò, and Singer (BMS) have introduced a free parameter, $\alpha_{K^{*}}[5]$, which weights the amplitude for $\mathrm{K}_{\mathrm{L}} \rightarrow \mathrm{K}^{*} \gamma \rightarrow(\rho, \omega, \phi) \gamma \rightarrow \gamma^{*} \gamma$ relative to that for $\mathrm{K}_{\mathrm{L}} \rightarrow\left(\pi^{0}, \eta, \eta^{\prime}\right) \rightarrow \gamma^{*} \gamma$. The first measurement of $\alpha_{\mathrm{K}^{*}}$ was reported by the NA31 collaboration at CERN in the $\mathrm{K}_{\mathrm{L}} \rightarrow \mathrm{e}^{+} \mathrm{e}^{-} \gamma$ channel [6]. Subsequent measurements were made at the Brookhaven National Laboratory (BNL experiment E845 [7]) and the Fermi National Accelerator Laboratory (FNAL experiment E799 [8]) in the electron and muon modes, respectively. Results on $\mathrm{K}_{\mathrm{L}} \rightarrow \mu^{+} \mu^{-} \gamma$ from a first run of the NA48 experiment at CERN were reported in 1997 [9]. Here we present the first results from $\mathrm{K}_{\mathrm{L}} \rightarrow \mathrm{e}^{+} \mathrm{e}^{-} \gamma$ in NA48 in a run which took place in 1997. Using a much larger data sample than was available in previous experiments, and with negligible background, $\alpha_{K^{*}}$ has been determined with improved accuracy. In addition, a rate for this decay has been measured in the experiment, which permits comparison of $\Gamma\left(\mathrm{K}_{\mathrm{L}} \rightarrow \mathrm{e}^{+} \mathrm{e}^{-} \gamma\right) / \Gamma\left(\mathrm{K}_{\mathrm{L}} \rightarrow \gamma \gamma\right)$ with the BMS model and calculation of a new $\mathrm{K}_{\mathrm{L}} \rightarrow \mathrm{e}^{+} \mathrm{e}^{-} \gamma$ branching ratio.

\section{Experimental setup}

The NA48 experiment at the CERN SPS is designed specifically to measure the direct $\mathrm{CP}$ violation parameter $\operatorname{Re}\left(\varepsilon^{\prime} / \varepsilon\right)$ using simultaneous beams of $\mathrm{K}_{\mathrm{L}}$ and $\mathrm{K}_{\mathrm{S}}$. To produce the $\mathrm{K}_{\mathrm{L}}$ beam, $450 \mathrm{GeV} / \mathrm{c}$ protons are extracted from the accelerator during $2.4 \mathrm{~s}$ every $14.4 \mathrm{~s}$ and $1.1 \times 10^{12}$ of these, spread over an effective burst length of about $1.7 \mathrm{~s}$, are delivered to a beryllium target. Using dipole magnets, charged particles are swept from a beam line aligned at $2.4 \mathrm{mrad}$ to the incident beam, and the resulting neutral beam, containing $2 \times 10^{7} \mathrm{~K}_{\mathrm{L}}$ per burst, has a divergence defined by collimators to $\pm 0.15 \mathrm{mrad}$. The fiducial volume for $\mathrm{K}_{\mathrm{L}}$ decay is contained in an evacuated steel cylinder $89 \mathrm{~m}$ long and $2.4 \mathrm{~m}$ in maximum diameter which begins $126 \mathrm{~m}$ downstream from the target. The cylinder is terminated at the downstream end by a window made of Kevlar-fibre composite of thickness $0.9 \mathrm{~mm}$ $\left(\sim 3 \times 10^{-3}\right.$ radiation lengths) and followed immediately by the main NA48 detector. This arrangement is illustrated in Fig. 1.

The sub-detectors which are used to record the $\mathrm{K}_{\mathrm{L}} \rightarrow \mathrm{e}^{+} \mathrm{e}^{-} \gamma$ are described below, in order of succession downstream of the decay region:

- A spectrometer consisting of a dipole magnet preceded and followed by two drift chambers within a helium-filled tank.

The $2.4 \mathrm{~m}$ square magnet aperture has a field integral of $0.85 \mathrm{Tm}$ which is uniform to about $\pm 5 \%$ in the fiducial region. Each chamber contains planes of parallel wires, staggered to resolve left-right ambiguities and oriented vertically and horizontally in all chambers, with additional planes at $\pm 45^{\circ}$ instrumented in all chambers except for the one directly behind the magnet. With singlecoordinate resolution of $100 \mu \mathrm{m}$, the spectrometer achieves a momentum resolution of $\Delta \mathrm{p} / \mathrm{p}=0.65 \%$ at $45 \mathrm{GeV} / \mathrm{c}$ and a measured invariant mass resolution of $2.6 \mathrm{MeV} / \mathrm{c}^{2}$ on kaons from $\mathrm{K}_{\mathrm{L}} \rightarrow \pi^{+} \pi^{-}$decays.

- A liquid krypton calorimeter ( $\mathrm{LKr}$ ) for measuring the energy, position, and timing of electromagnetic showers initiated by electrons and photons.

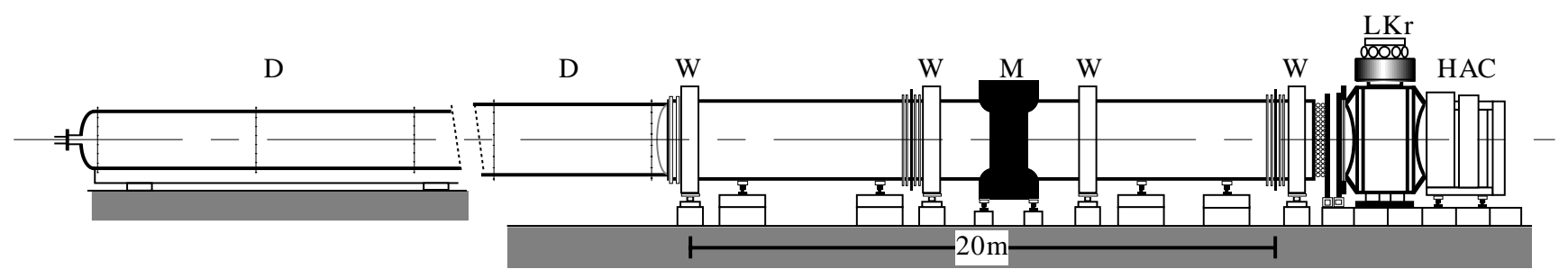

Fig. 1. Side-view of elements of the NA48 detector which are most relevant for the present analysis. Particles in the neutral beam travel along the axis from left to right, traversing the $89 \mathrm{~m}$ long evacuated fiducial volume for $\mathrm{K}_{\mathrm{L}}$ decay (D). This is followed by the spectrometer containing a dipole magnet $(\mathrm{M})$ and wire drift chambers $(\mathrm{W})$, the liquid krypton calorimeter (LKr), and the hadron calorimeter (HAC). The fiducial volume is separated from the spectrometer by a thin Kevlar window and the beam subsequently pa sses through the remaining detector elements in an evacuated tube. 
Krypton is chosen as the active medium because of its high density and small Molière radius $(4.7 \mathrm{~cm})$ which results in well-contained (and hence well-separated) electromagnetic showers. About $9 \mathrm{~m}^{3}$ of liquid krypton are maintained in a cryostat at $121 \mathrm{~K}$. The calorimeter is divided into 13212 read-out cells by $\mathrm{Cu}$-Be ribbons stretched longitudinally. Each cell is $2 \mathrm{~cm} \times 2 \mathrm{~cm}$ in crosssection and $127 \mathrm{~cm}$ long (27 radiation lengths in liquid krypton). The ionization signal from each cell is amplified, shaped, and digitized by $40 \mathrm{MHz}$ 10-bit FADC's. Gain-switching increases the dynamic range to approximately 14 bits. Special attention was paid to precise charge calibration, with data taken every burst. A resolution of

$$
\frac{\sigma}{\mathrm{E}}=\frac{0.035}{\sqrt{\mathrm{E}}} \oplus \frac{0.110}{\mathrm{E}} \oplus 0.006,
$$

with $\mathrm{E}$ in $\mathrm{GeV}$, was determined using electrons from $\mathrm{K}_{\mathrm{L}} \rightarrow \pi^{ \pm} \mathrm{e}^{\mp} \mathrm{V} \quad\left(\mathrm{K}_{\mathrm{e} 3}\right)$ decays, simulation studies, and knowledge of noise and filtering sensitivity.

- A hadron calorimeter composed of 48 steel plates, each $24 \mathrm{~mm}$ thick, interleaved with scintillator and used for particle identification.

The wire chambers are used to measure the electron momenta and to reconstruct the position of the decay vertex, while the $\mathrm{LKr}$ measures impact positions and energies of electrons and photons. Signals from the hadron calorimeter were used in trigger formation and for cut efficiency studies.

A more detailed and comprehensive account of the NA48 beam and detector is to be published [10].

\section{Trigger and data processing}

The neutral trigger in NA48 is designed to accept $\mathrm{K}_{\mathrm{L}} \rightarrow \mathrm{e}^{+} \mathrm{e}^{-} \gamma, \mathrm{K}_{\mathrm{L}} \rightarrow \pi^{0} \pi_{\text {Dalitz }}^{0}$ and other rare decay channels as well as to signal the presence of a likely $\mathrm{K} \rightarrow \pi^{0} \pi^{0}$ decay for which it was primarily conceived and for which its efficiency is $99.9 \%$. The trigger utilizes analogue signals from the $\mathrm{LKr}$ calorimeter which are digitized and summed to yield energies in columns and rows each two cells wide. This information is used to look for peaks in horizontal and vertical projections, to contribute to a total energy sum, and to form the first and second moments of energy in both projections. Look-up tables enable the rapid calculation of signal moments and estimation of the kaon decay vertex based on calorimeter information only. The calorimeter signals were monitored continuously and a new trigger decision issued every 25 nsec.
After decoding of the raw data and the application of calibration constants, reconstruction of showers in the $\mathrm{LKr}$ proceeds by searching for local maxima in energy deposition and by iteratively exploring energy deposition in the vicinity of these maxima in the light of expected shower profiles. Corrected positions, shower sizes, and energies are calculated, after which small corrections are made, taking into account leakage into the beam pipe, dead cells, and calorimeter structure.

During the experimental run, 23.5 terabytes of raw data were recorded by NA48. Reconstructed output was stored in a highly compressed format amenable to fast $\mathrm{I} / \mathrm{O}$. The progression from raw data to compact data involved partial reconstruction followed by filtering of the data to form appropriate streams for the analysis of neutral or charged two-pion final states, or for rare decays. At this point, full reconstruction and compaction were carried out. The resulting size of a compact event was typically 1.5 kbytes compared to about 20 kbytes for an event in raw data.

\section{Data analysis}

For final states containing only photons or a mixture of electrons and photons, the evaluation of first and second moments of cluster energy in the LKr with respect to the beam axis is especially useful. The first moment (the "centre of gravity") is typically less than $4.5 \mathrm{~cm}$ for decays in which no energy is lost from the calorimeter and is unaffected by the presence of the magnet. The second moment and the constraint that the decay should come from a kaon are used to obtain the axial vertex position, $\mathrm{z}$, of the decay using only shower positions and energies. In the case of electrons, an offline correction to $\mathrm{z}$ is applied based on extrapolation of the trajectories upstream of the spectrometer magnet.

In events containing electron pairs, vertices are also determined using trajectory information only. The added constraint that the kaon decay take place on the line joining the centre of gravity to the target is used for pairs with small opening angles.

Proper lifetime for kaon decay in this analysis is measured in units of the $\mathrm{K}_{\mathrm{S}}$ lifetime $\tau_{\mathrm{Ks}}$, which is the natural scale for the $\varepsilon^{\prime} / \varepsilon$ measurement in NA48. As will be seen, background events do not deposit all their energy in the LKr. As a consequence, vertices for purely neutral background decays are reconstructed too far downstream and the kaon energy is underestimated, so that the resulting calculated lifetime tends to be overestimated, facilitating rejection.

The neutral trigger applied the following cuts to the data online:

$-\leq 5$ peaks in each projection; 
- Total energy in the $\mathrm{LKr}>50.0 \mathrm{GeV}$;

- centre of gravity < $15 \mathrm{~cm}$;

- Lifetime uncorrected for deflection in the magnet $<5.5 \tau_{\mathrm{Ks}}$.

The efficiency of the neutral trigger was measured as a function of vertex position calculated offline but uncorrected for the deflection of charged particles in the magnetic field. This measurement was carried out using a special Dalitz trigger which accepted $\mathrm{K}_{\mathrm{L}} \rightarrow \mathrm{e}^{+} \mathrm{e}^{-} \gamma$ decays within a loose cut corresponding to $8.5 \tau_{\mathrm{Ks}}$ from the start of the fiducial region. The efficiency was found to be (99.6 $\pm 0.2) \%$ and flat up to about $4 \tau_{\mathrm{Ks}}$. from where there is a decrease to $85 \%$ at $6 \tau_{\mathrm{Ks}}$.

Further cuts were applied at the filtering stage:

- Exactly three clusters were required;

- Two tracks in the spectrometer were required, loosely associated with clusters in the $\mathrm{LKr}$ having $\mathrm{E}($ cluster $) / \mathrm{p}($ track $)>0.85$;

- Corrected lifetime $<8.0 \tau_{\mathrm{Ks}}$ (chosen loose to increase the number of Dalitz trigger events used in trigger efficiency studies);

- centre of gravity < $10 \mathrm{~cm}$;

- Energy in the hadron calorimeter $<8 \mathrm{GeV}$.

Offline cuts to enhance the signal-to-background ratio were made as follows:

- All clusters in the LKr were required to be sufficiently far from the beam pipe, the outer radius, and dead cells to ensure negligible energy loss.

- The separation between cluster centres was required to be $>5 \mathrm{~cm}$, a cut which ensures good reconstruction efficiency as confirmed by Monte Carlo studies.

- The energy of each cluster was required to be between 3 and $120 \mathrm{GeV}$. The lower limit is well above the level of detector noise $(\sim 110 \mathrm{MeV}$ per cluster). Moreover the efficiency for reconstruction of photons is known from separate studies to be $>99.7 \%$ for energies above $3 \mathrm{GeV}$. The upper limit is within the dynamic range of the $\mathrm{LKr}$ signal digitizing electronics.

- The two tracks in the spectrometer were required to be separated by $\geq 2 \mathrm{~cm}$ in the first drift chamber. This cut is effective in rejecting $\mathrm{K}_{\mathrm{L}} \rightarrow \gamma \gamma$ events in which one of the photons converts before the magnet.

- Electron candidates were identified by requiring that cluster centres in the $\mathrm{LKr}$ be within $1.5 \mathrm{~cm}$ of calculated shower maxima based upon the extrapolation of each track. The efficiency of this procedure was measured to be $(99.7$ $\pm 0.1) \%$ using electrons in a sample of $\mathrm{K}_{\mathrm{e} 3}$ events.

- As a further means of rejecting pion showers, it was required that E/p lie between 0.9 and 1.1, and that each track deposit less than $2.5 \mathrm{GeV}$ in the hadron calorimeter. The efficiency of the E/p cut was thoroughly studied as a function of momentum, again using $\mathrm{K}_{\mathrm{e} 3}$ events. Generally it was $\geq 95 \%$ efficient over the accepted range of momentum. In the same study, the efficiency for accepting electrons as a result of the energy cut in the hadron calorimeter was found to be $(99.4 \pm 0.2) \%$.

The cluster not associated with a track was identified as the photon candidate. Using the average time of the two electron candidates to define the "event time", the timing of the photon candidate was required to agree to within $\pm 2.5 \mathrm{nsec}$. This requirement minimizes background from $\mathrm{K}_{\mathrm{e} 3}$ events (with the pion mis-identified as an electron) accompanied by an accidental photon.

Several online cuts were tuned in the light of acceptance considerations: the centre-of-gravity cut was reduced to $5 \mathrm{~cm}$ and the total energy in the $\mathrm{LKr}$ required to be between 60.0 and $190.0 \mathrm{GeV}$. Further, a radial vertex cut demanded that decays be consistent with occurrence in the $\mathrm{K}_{\mathrm{L}}$ beam, rather then in the adjacent $\mathrm{K}_{\mathrm{S}}$ beam used in simultaneous $\varepsilon^{\prime} / \varepsilon$ running. For this particular cut, the difficulty of vertex determination posed by tracks at small opening angles was mitigated in the following way: First the axial vertex coordinate $\mathrm{z}$ was calculated using shower positions and energies only. Then the transverse position of each track at this $\mathrm{z}$ was determined and the average of these taken to be the $x$ and $y$ coordinates of the vertex. In addition, the conversion of one photon from $\mathrm{K}_{\mathrm{S}} \rightarrow \pi^{0} \pi^{0}$ in a veto counter defining the start of the $\mathrm{K}_{\mathrm{S}}$ fiducial region, followed by the loss of two more photons, could (though at a very low rate), mimic $\mathrm{K}_{\mathrm{S}} \rightarrow \pi^{0} \pi_{\text {Dalitz }}^{0}$, itself a source of background to be discussed. It was therefore required that there be no in-time activity in this veto counter.

The fiducial volume was defined from an axial position $750 \mathrm{~cm}$ downstream of the $\mathrm{K}_{\mathrm{S}}$ target to $3150 \mathrm{~cm}$. This latter position was chosen to maximize the number of events while correcting safely any bias caused by the neutral trigger lifetime cut. Within the fiducial volume, vertices were determined with a typical axial resolution of $\pm 70 \mathrm{~cm}$.

After the application of these cuts, 19497 $\mathrm{K}_{\mathrm{L}} \rightarrow \mathrm{e}^{+} \mathrm{e}^{-} \gamma$ candidates remain. In Fig. 2 the mass, $\mathrm{m}_{\mathrm{ee} \gamma}$, calculated using the vertex and positions (corrected for bending of the electron trajectories in the magnetic field) and energies of the clusters in the $\mathrm{LKr}$, is plotted against the scaled invariant mass of the electron pair squared, $\left(\mathrm{m}_{\mathrm{ee}} / \mathrm{m}_{\mathrm{K}}\right)^{2}$, calculated using the momenta and trajectories of the electrons in the drift chambers. A clear band is seen around the kaon mass. Background events are distributed in the lower part of the plot and most were identified as $\mathrm{K}_{\mathrm{e} 3}$ with external bremsstrahlung. The signal region is defined by dashed lines which correspond to $5 \sigma$ limits on the 
detector resolution. Within the signal region lie 6864 events.

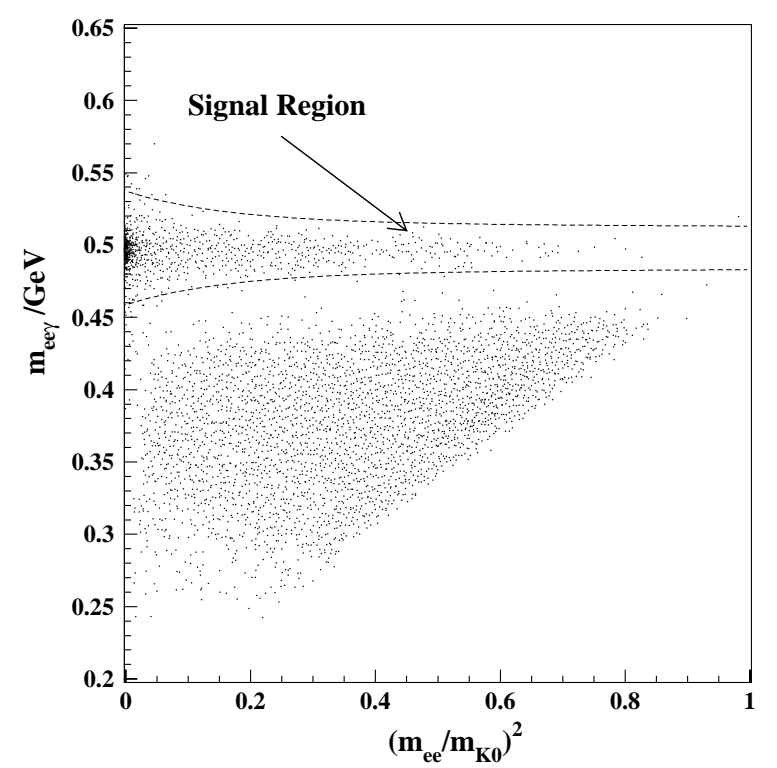

Fig. 2. Scatter plot of $\mathrm{e}^{+} \mathrm{e} \gamma$ mass versus $\mathrm{m}_{\mathrm{ee}}^{2} / \mathrm{m}_{\mathrm{K}^{0}}^{2}$. The dashed lines define the signal region.

A comprehensive study of possible sources of background in the signal region confirmed that the cuts described above had rendered the following backgrounds insignificant:

- $\mathrm{K}_{\mathrm{L}} \rightarrow \gamma \gamma$ with conversion of a photon before the spectrometer magnet. The required separation of two tracks in the first chamber eliminated background from this source.

- $\mathrm{K}_{\mathrm{L}} \rightarrow \pi^{0} \pi^{0} \pi_{\text {Dalitz }}^{0}$ with missing photons. Here, missing energy caused the vertex of the apparent $\mathrm{K} \rightarrow \mathrm{e}^{+} \mathrm{e}^{-} \gamma$ to fall far outside the accepted range.

- $\mathrm{K}_{\mathrm{e} 3}$ with the pion mis-identified as an electron and external bremsstrahlung; $\mathrm{K}_{\mathrm{e} 3}$ with the pion being misidentified as a photon + electron owing to a hadronic shower fluctuation; $\mathrm{K}_{\mathrm{e} 3}$ with internal bremsstrahlung and mis-identification of the pion as an electron.

- All sources of background requiring the misidentification of two pions, such as $\mathrm{K}_{\mathrm{L}} \rightarrow \pi^{+} \pi^{-}$and $\mathrm{K}_{\mathrm{L}} \rightarrow \pi^{+} \pi^{-} \pi^{0}$.

Backgrounds from three other sources are large enough to be estimated in a practical way though they remain extremely small fractions of the signal. These are discussed below and the numbers of events which remain in the signal region from these sources are summarized in Table I. Backgrounds from the first two sources were obtained from Monte Carlo studies, but have been cross-checked with data. Background from $\mathrm{K}_{\mathrm{e} 3(\mathrm{\gamma acc})}$ was estimated using data alone:
- $\mathrm{K}_{\mathrm{L}} \rightarrow \pi^{0} \pi_{\text {Dalitz }}^{0}$ with missing photons. Since there is lost energy, events from this source tend to be reconstructed with mass below that of the kaon.

- $\mathrm{K}_{\mathrm{S}} \rightarrow \pi^{0} \pi_{\text {Dalitz }}^{0}$ with missing photons. The lost energy in these events leads to an incorrect determination of both the axial and transverse coordinates of the calculated vertex position. As a consequence, some of these events appear to originate in the $\mathrm{K}_{\mathrm{L}}$ beam. Here the fact that most decays occur early in the fiducial region makes the trigger less effective at removal with a lifetime cut. Moreover, it is observed in Monte Carlo studies that using the centre-of-gravity-to- $\mathrm{K}_{\mathrm{L}}$-target line to constrain the $\mathrm{z}$ vertex shifts the reconstructed mass to values in the vicinity of the kaon mass, despite the missing energy.

- $\mathrm{K}_{\mathrm{e} 3}$ accidentally in coincidence with a photon in the LKr. The effect of this source was estimated by removing the requirement that the photon cluster be in time with the electron clusters. The resulting distribution was flat in time as expected, and a control window away from the signal region yielded the amount of contamination.

\begin{tabular}{|c|c|}
\hline Background & No. of Events \\
\hline $\mathrm{K}_{\mathrm{L}} \rightarrow \pi^{0} \pi_{\text {Dalitz }}^{0}$ & $0.3 \pm 0.2$ \\
\hline $\mathrm{K}_{\mathrm{S}} \rightarrow \pi^{0} \pi_{\text {Dalitz }}^{0}$ & $7.2 \pm 1.2$ \\
\hline $\mathrm{K}_{\mathrm{e} 3(\gamma \mathrm{acc})}$ & $2.9 \pm 0.6$ \\
\hline Total & $10.4 \pm 1.4$ \\
\hline
\end{tabular}

Table I. Background events remaining in the $\mathrm{K}_{\mathrm{L}} \rightarrow \mathrm{e}^{+} \mathrm{e}^{-} \gamma$ signal region.

\section{Normalization}

The normalization chosen is $\mathrm{K}_{\mathrm{L}} \rightarrow \pi^{0} \pi_{\text {Dalitz }}^{0}$ for which the effects of any inefficiency in the drift chambers and of electron identification largely divide out in the ratio of signal and normalization.

Using cuts analogous to those employed for selecting $\mathrm{K}_{\mathrm{L}} \rightarrow \mathrm{e}^{+} \mathrm{e}^{-} \gamma$ decays, $4084 \mathrm{~K}_{\mathrm{L}} \rightarrow \pi^{0} \pi_{\text {Dalitz }}^{0}$ events were found. Background predominantly is due to $\mathrm{K}_{\mathrm{L}} \rightarrow \pi^{0} \pi^{0} \pi_{\text {Dalitz }}^{0}$ with lost photons and $\mathrm{K}_{\mathrm{S}} \rightarrow \pi^{0} \pi_{\text {Dalitz }}^{0}$ from the adjacent beam, with badly reconstructed vertices. Studies of the data indicate $12 \pm 2$ residual events in the $\mathrm{K}_{\mathrm{L}} \rightarrow \pi^{0} \pi_{\text {Dalitz }}^{0}$ signal from the former process and Monte Carlo results suggest another $12 \pm 2$ events from the latter.

\section{Results and discussion}

\section{a. Measurement of $\alpha_{K^{*}}$}

In the absence of radiative corrections, the form factor $\mathrm{f}(\mathrm{x})$ parameterizes the differential decay spectrum as follows [11]: 


$$
\Gamma_{\gamma}^{-1} \frac{\mathrm{d} \Gamma}{\mathrm{dx}}=\frac{2 \alpha}{3 \pi} \frac{\left(1-\mathrm{x}^{3}\right)}{\mathrm{x}}\left[1+\frac{2 \mathrm{~m}_{\mathrm{e}}^{2}}{\mathrm{xm}_{\mathrm{K}}^{2}}\right]\left[1-\frac{4 \mathrm{~m}_{\mathrm{e}}^{2}}{\mathrm{xm}_{\mathrm{K}}^{2}}\right]^{1 / 2}|\mathrm{f}(\mathrm{x})|^{2},
$$

where $x=m_{\text {ee }}^{2} / m_{K}^{2}$. In numerical form, $f(x)$ as a function of $\alpha_{\mathrm{K}}{ }^{*}$ can be expressed in the BMS model [7]:

$$
\begin{aligned}
& f(x)=\frac{1}{1-0.418 x}+\frac{2.5 \alpha_{K^{*}}}{1-0.311 x}\left[\frac{4}{3}-\frac{1}{1-0.418 x}\right. \\
& \left.-\frac{1}{9(1-0.405 x)}-\frac{2}{9(1-0.238 x)}\right] .
\end{aligned}
$$

With the inclusion of QED radiative processes, the spectrum is no longer described correctly as a product of $\mathrm{f}(\mathrm{x})$ and phase space. Therefore for the determination of $\alpha_{\mathrm{K}^{*}}$ ten Monte Carlo samples were generated and a full detector simulation performed. Each sample contained $\sim 10^{5}$ accepted events. For calculation of radiative corrections, the method and code of H.B. Greenlee [12] have been adapted.

A $\chi^{2}$ test between the data (taking account of background) and the Monte Carlo events was performed on the $\mathrm{m}_{\mathrm{ec}}$ distributed in twelve bins, for the ten values of $\alpha_{\mathrm{K}^{*}}$. The result is shown in Fig.3 where a typical error of \pm 1.6 is assigned to each point, based on studies with independent samples. The minimum of a parabolic fit over the range $-0.55<\alpha_{\mathrm{K}^{*}}<-0.19$ yielded a value of $\alpha_{\mathrm{K}^{*}}$ of $-0.37 \pm 0.06$, where the error given is that for $\chi^{2}$ to increase by one. In comparison with this statistical uncertainty, the error on the fit and the systematic error found by varying the range are both negligible.

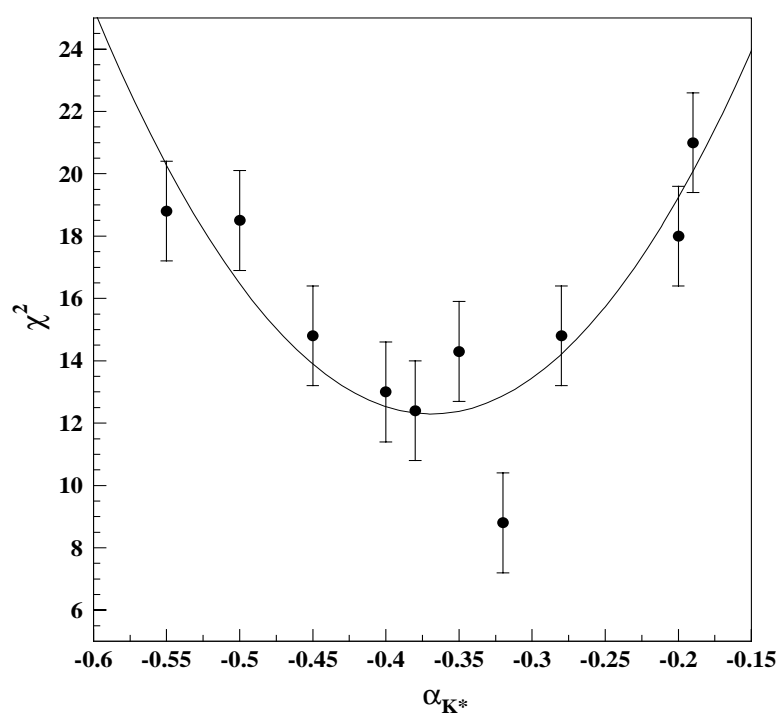

Fig. 3. $\chi^{2}$ vs. $\alpha_{\mathrm{K}}$ * between data and Monte Carlo events for the $\mathrm{m}_{\mathrm{ee}}$ distribution.

Without radiative corrections, the value of $\alpha_{\mathrm{K}}$ *above would be $-0.15 \pm 0.05$. This difference arises because at high $\mathrm{m}_{\mathrm{ee}}$ the probability of emission of internal bremsstrahlung increases and the resulting eery events are lost from our sample. The effect should not, however, be interpreted as contributing to the systematic error on the result as the correction procedure is well-understood.

Since the analysis relies on the proper understanding of the Monte Carlo simulation, comparisons between data and Monte Carlo are made wherever possible. Figures 4 and 5 show typical comparisons between the $\mathrm{K}_{\mathrm{L}} \rightarrow \mathrm{e}^{+} \mathrm{e}^{-} \gamma$ data and Monte Carlo, here for the kaon energy spectrum and the $\mathrm{z}$ vertex, respectively.

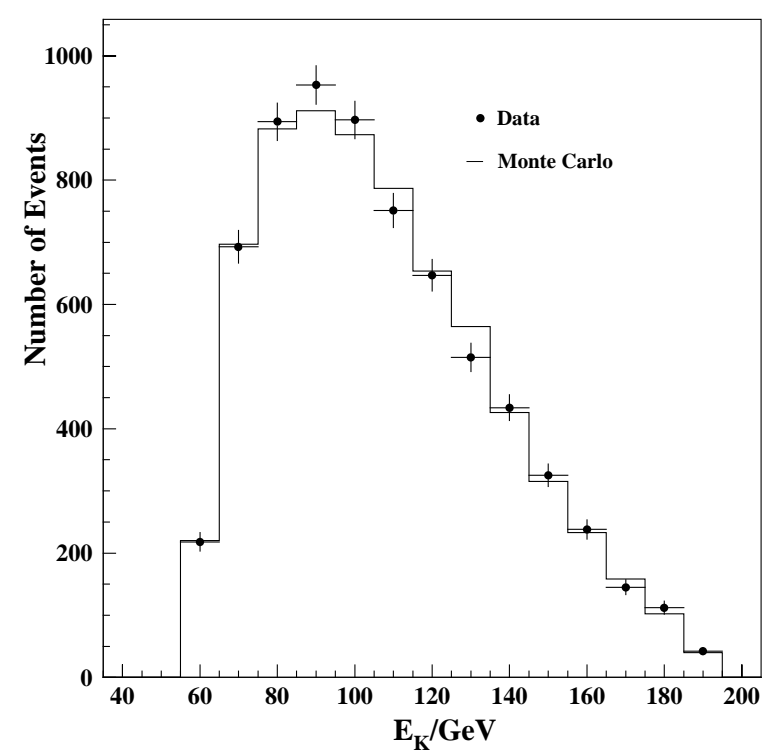

Fig. 4. Comparison between data and Monte Carlo in reconstructed kaon energy from $\mathrm{K}_{\mathrm{L}} \rightarrow \mathrm{e}^{+} \mathrm{e}^{-} \gamma$.

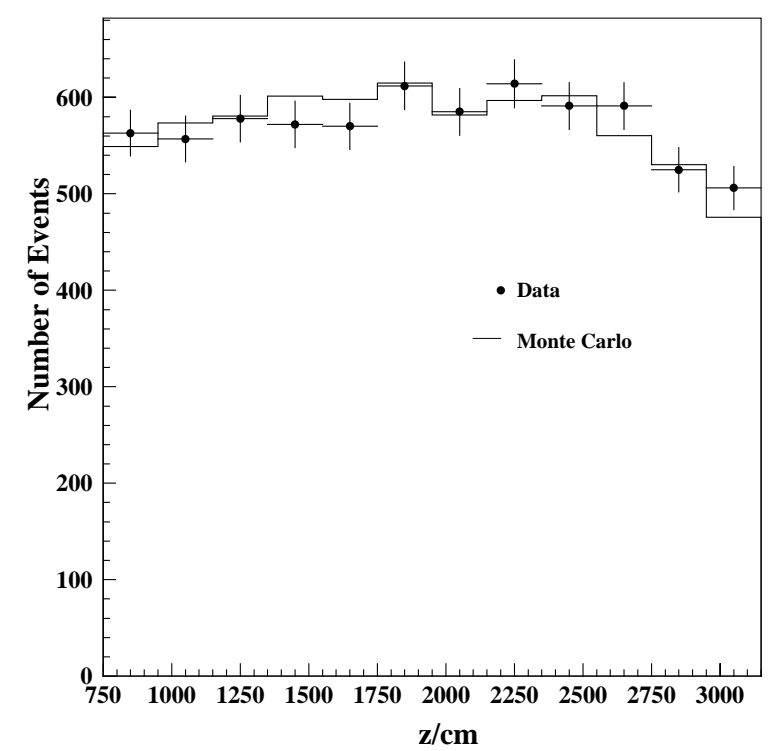

Fig. 5. Comparison between data and Monte Carlo in the reconstructed $\mathrm{z}$ vertex position from $\mathrm{K}_{\mathrm{L}} \rightarrow \mathrm{e}^{+} \mathrm{e}^{-} \gamma$.

No significant systematic variation of $\alpha_{\mathrm{K}}{ }^{*}$ is seen as a function of $\mathrm{z}$ vertex position, kaon energy or track separation in the first drift chamber. A small observed 
variation in the value of $\alpha_{\mathrm{K}^{*}}$ as a function of minimum electron energy may be significant and attributable to tracking inefficiency at low momentum where larger deflections in the spectrometer occur. A consideration of this possibility in the light of track reconstruction studies suggests a correction to $\alpha_{\mathrm{K}} *$ of $+2.9 \%$ with an uncertainty of $\pm 2.9 \%$.

The effect on $\alpha_{K}{ }^{*}$ of a $\pm 2 \%$ variation in the kaon energy spectrum of the Monte Carlo as compared with data was found to be negligible to within ${ }_{-0.6}^{+0.0} \%$.

An uncertainty of $\pm 1.8 \%$ is attributable to limitation in the knowledge of $\mathrm{LKr}$ response to low energy photons produced as internal bremsstrahlung from the decay electrons. This result arises from a study of how Monte Carlo and data might reasonably differ for cluster energies between a common threshold of $0.75 \mathrm{GeV}$ and a level of $3.0 \mathrm{GeV}$ (the normal minimum level for $\varepsilon^{\prime} / \varepsilon$ data) where comparison is straightforward and agreement is good.

The study of $\alpha_{K^{*}}$ values, as cuts on maximum cluster energy, E/p cut, neutral trigger efficiency, and radial vertex cut were varied in Monte Carlo, showed that no significant correction was needed.

Table II summarizes systematic correction and errors in the measurement of $\alpha_{\mathrm{K}^{*}}$.

\begin{tabular}{|l|c|c|}
\hline Effect & Correction & $\begin{array}{c}\text { Systematic } \\
\text { Error }\end{array}$ \\
\hline $\begin{array}{l}\text { Tracking inefficiency at } \\
\text { low momentum. }\end{array}$ & $+2.9 \%$ & $\pm 2.9 \%$ \\
\hline Kaon energy spectrum & - & ${ }_{-0.6}^{+0.0} \%$. \\
\hline $\begin{array}{l}\text { Cut on maximum cluster } \\
\text { energy. }\end{array}$ & - & ${ }_{-0}^{+4} \%$ \\
\hline $\begin{array}{l}\text { Uncertainty in effect of } \\
\text { E/p cut }\end{array}$ & - & ${ }_{-1.9}^{+0.9} \%$ \\
\hline Neutral trigger efficiency & - & ${ }_{-0.3}^{+0.7} \%$ \\
\hline Radial vertex cut & - & ${ }_{-0.0}^{+0.9} \%$ \\
\hline $\begin{array}{l}\text { LKr response at low } \\
\text { energy }\end{array}$ & - & $\pm 1.8 \%$ \\
\hline
\end{tabular}

Table II. Systematic effects in the measurement of $\alpha_{\mathrm{K}}{ }^{*}$.

Applying the indicated correction and combining the systematic errors from Table II in quadrature yields the corrected value

$$
\alpha_{\mathrm{K}^{*}}=-0.36 \pm 0.06 \pm 0.02,
$$

where the first error is that for the $\chi^{2}$ to increase by one and the second is systematic.

Data are displayed in Fig. 6 in bins of $\mathrm{x}=\left(\mathrm{m}_{\mathrm{ee}} / \mathrm{m}_{\mathrm{K} 0}\right)^{2}$ together with the results of Monte Carlo simulations with $\alpha_{\mathrm{K}^{*}}=-0.36$ and with $\mathrm{f}(\mathrm{x})=1$, respectively. Each simulation contains appropriate radiative corrections.

The theoretical prediction of the BMS model is in agreement with this result, as are previous measurements based on smaller numbers of $\mathrm{K}_{\mathrm{L}} \rightarrow \mathrm{e}^{+} \mathrm{e}^{-} \gamma$ events [5,6,7]. The two measurements of $\alpha_{\mathrm{K}}$ * from $\mathrm{K}_{\mathrm{L}} \rightarrow \mu^{+} \mu^{-} \gamma$ favour negative values smaller in magnitude $[8,9]$.

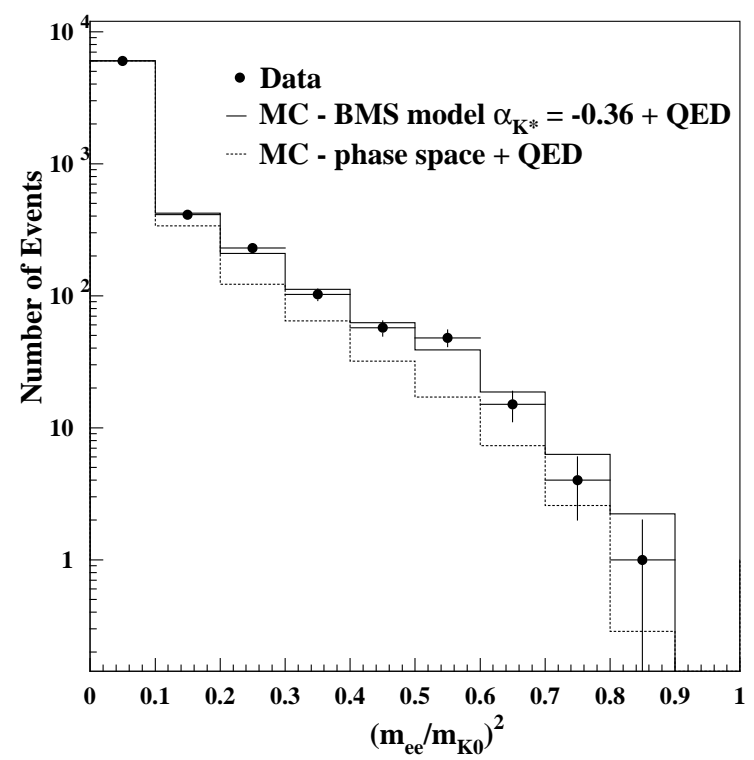

Fig. 6. Comparison of data variation in $\mathrm{x}=\left(\mathrm{m}_{\mathrm{ee}} / \mathrm{m}_{\mathrm{K} 0}\right)^{2}$ with Monte Carlo samples generated using $\alpha_{\mathrm{K}^{*}}=-0.36$ (solid line) and with $\mathrm{f}(\mathrm{x})=1$ (dashed line, normalized to the data in the first bin). Both Monte Carlo calculations contain appropriate radiative corrections.

\section{b. Branching ratio}

After correction for background there remain 6854 $\mathrm{K}_{\mathrm{L}} \rightarrow \mathrm{e}^{+} \mathrm{e}^{-} \gamma$ candidates for which Monte Carlo calculations show an acceptance of $(15.65 \pm 0.07) \%$ and $4060 \mathrm{~K}_{\mathrm{L}} \rightarrow \pi^{0} \pi_{\text {Dalitz }}^{0}$ events with an acceptance of $(4.30 \pm 0.03) \%$. The uncorrected ratio of $\mathrm{K}_{\mathrm{L}} \rightarrow \mathrm{e}^{+} \mathrm{e}^{-} \gamma$ to $\mathrm{K}_{\mathrm{L}} \rightarrow \pi^{0} \pi_{\text {Dalitz }}^{0}$ rates is therefore $0.464 \pm 0.010$ before accounting for systematic effects as follows:

- The kaon energy spectrum in the Monte Carlo agrees with that found in the data to $\pm 2 \%$. The effect of this on the ratio is negligible to within an estimated ${ }_{-0.6}^{+0.0} \%$.

- The ratio was not significantly affected as the cut on track separation at the first drift chamber was varied.

- A study of the stability of the ratio was made as a function of acceptance at the LKr. Variation of the cuts on separation, energy, and radial positions of clusters had no significant effect, nor was there any significant difference between charged and neutral energy scales. On the other hand, a slight variation of the ratio with cuts on invariant mass combinatorics in the $\pi^{0} \pi_{\text {Dalitz }}^{0}$ final states led to an estimate of $\pm 1.4 \%$ on the uncertainty in the ratio, though no significant correction is required.

- A small drop in chamber efficiency for tracks close to the beam pipe was estimated in Monte Carlo studies to result in a $1 \%$ loss of $\mathrm{K}_{\mathrm{L}} \rightarrow \pi^{0} \pi_{\text {Dalitz }}^{0}$ events and a $0.4 \%$ 
loss of $\mathrm{K}_{\mathrm{L}} \rightarrow \mathrm{e}^{+} \mathrm{e}^{-} \gamma$. A correction of $-0.6 \%$ (with an uncertainty of $\sim 20 \%$ ) was therefore applied to the ratio.

- Variation within errors of the E/p cut used in the Monte Carlo led to small differential acceptances suggesting an uncertainty of ${ }_{-0.0}^{+0.3 \%}$ in the ratio from this source.

- The acceptance for $\mathrm{K}_{\mathrm{L}} \rightarrow \mathrm{e}^{+} \mathrm{e}^{-} \gamma$ exhibits an approximately linear $\pm 2.0 \%$ variation as a function of $\alpha_{\mathrm{K}}{ }^{*}$ in the range 0.0 to -0.4 . As the form factor parameter has been measured to be $-0.36 \pm 0.06$, an associated systematic error of $\pm 0.3 \%$ is ascribed to the ratio.

- In studies of data taken with the Dalitz trigger, the neutral trigger was found to be about $1 \%$ less efficient for $\mathrm{K}_{\mathrm{L}} \rightarrow \pi^{0} \pi_{\text {Dalitz }}^{0}$ events than for $\mathrm{K}_{\mathrm{L}} \rightarrow \mathrm{e}^{+} \mathrm{e}^{-} \gamma$. This is not surprising at the $1 \%$ level as differences in electron momenta and in cluster energies and angles between the two decays produce corresponding differences in uncorrected vertex positions and resolutions, and cluster merging. Further, the probabilities of cluster-splitting in the $\mathrm{LKr}$, determined in $\mathrm{K}_{\mathrm{S}} \rightarrow \pi^{0} \pi^{0}$ studies, suggest the need for a $(0.22 \pm 0.01) \%$ correction in the ratio owing to loss of $\mathrm{K}_{\mathrm{L}} \rightarrow \pi^{0} \pi_{\text {Dalitz }}^{0}$ events arising from the peaks condition in the neutral trigger.

- The largest correction to the ratio arises from consideration of the $\mathrm{K}_{\mathrm{S}}$ component in the $\mathrm{K}_{\mathrm{L}}$ beam. This is produced by interference in the fiducial region which is located at a distance from the $\mathrm{K}_{\mathrm{L}}$ target greater than $\sim 15 \mathrm{~K}_{\mathrm{S}}$ lifetimes for a typical kaon. The consequent excess of $\pi^{0} \pi_{\text {Dalitz }}^{0}$ decays was studied in comparisons between data and Monte Carlo simulations resulting in a correction of $+(3.9 \pm 0.4) \%$ to the ratio.

These results are summarized in Table III.

\begin{tabular}{|c|c|c|}
\hline Effect & Correction & $\begin{array}{c}\text { Systematic } \\
\text { Error }\end{array}$ \\
\hline Kaon energy spectrum & - & ${ }_{-0.6}^{+0.0} \%$ \\
\hline$\pi^{0} \pi_{\text {Dalitz }}^{0}$ cuts & - & $\pm 1.4 \%$ \\
\hline $\begin{array}{l}\text { Tracking inefficiency } \\
\text { close to beam pipe }\end{array}$ & $-0.6 \%$ & $\pm 0.1 \%$ \\
\hline Uncertainty in $\mathrm{E} / \mathrm{p}$ cut & - & $\begin{array}{l}+0.3 \% \\
-0.0\end{array}$ \\
\hline $\begin{array}{l}\text { Effect of } \\
\alpha_{\mathrm{K}} * \text { uncertainty }\end{array}$ & - & $\pm 0.3 \%$ \\
\hline $\begin{array}{l}\text { Neutral trigger efficiency } \\
\text { for } \mathrm{K}_{\mathrm{L}} \rightarrow \pi^{0} \pi_{\text {Dalitz }}^{0}\end{array}$ & $-1.1 \%$ & $\pm 1.1 \%$ \\
\hline $\begin{array}{l}\text { Neutral trigger peaks } \\
\text { condition }\end{array}$ & $-0.2 \%$ & - \\
\hline $\mathrm{K}_{\mathrm{S}}$ component in beam & $+3.9 \%$ & $\pm 0.4 \%$ \\
\hline
\end{tabular}

Table III. Corrections and systematic error on the measurement of the ratio $\mathrm{K}_{\mathrm{L}} \rightarrow \mathrm{e}^{+} \mathrm{e}^{-} \gamma$ to $\mathrm{K}_{\mathrm{L}} \rightarrow \pi^{0} \pi_{\text {Dalitz }}^{0}$.
Application of the corrections and propagation of the systematic errors in quadrature yield

$$
\frac{\Gamma\left(\mathrm{K}_{\mathrm{L}} \rightarrow \mathrm{e}^{+} \mathrm{e}^{-} \gamma\right)}{\Gamma\left(\mathrm{K}_{\mathrm{L}} \rightarrow \pi^{0} \pi_{\text {Dalitz }}^{0}\right)}=0.473 \pm 0.010 \pm 0.009
$$

where the first error is statistical and the second systematic.

Using measured values of the branching ratio of $\pi^{0} \rightarrow \mathrm{e}^{+} \mathrm{e}^{-} \gamma[13]$ and $\Gamma\left(\mathrm{K}_{\mathrm{L}} \rightarrow \gamma \gamma\right) / \Gamma\left(\mathrm{K}_{\mathrm{L}} \rightarrow \pi^{0} \pi^{0}\right)$ [14], one can calculate the ratio

$$
\frac{\Gamma\left(\mathrm{K}_{\mathrm{L}} \rightarrow \mathrm{e}^{+} \mathrm{e}^{-\gamma}\right)}{\Gamma\left(\mathrm{K}_{\mathrm{L}} \rightarrow \gamma\right)}=(17.9 \pm 0.4 \pm 0.3 \pm 0.5) \times 10^{-3},
$$

where the first two errors are statistical and systematic respectively, and the last reflects uncertainty in the intermediate value used in the calculation. The theoretical prediction of the BMS model for this ratio is

$$
\Gamma\left(\mathrm{K}_{\mathrm{L}} \rightarrow \mathrm{e}^{+} \mathrm{e}^{-} \gamma\right) / \Gamma\left(\mathrm{K}_{\mathrm{L}} \rightarrow \gamma \gamma\right)=16.8 \times 10^{-3}
$$

using the value of $\alpha_{\mathrm{K}}{ }^{*}$ measured in this analysis.

Finally, using the measured branching ratio of $\mathrm{K}_{\mathrm{L}} \rightarrow \pi^{0} \pi^{0}[15]$, we obtain

$$
\frac{\Gamma\left(\mathrm{K}_{\mathrm{L}} \rightarrow \mathrm{e}^{+} \mathrm{e}^{-} \gamma\right)}{\Gamma\left(\mathrm{K}_{\mathrm{L}} \rightarrow \text { all }\right)}=(1.06 \pm 0.02 \pm 0.02 \pm 0.04) \times 10^{-5}
$$

where the last error is dominated by uncertainties in the $\pi^{0} \rightarrow \mathrm{e}^{+} \mathrm{e}^{-} \gamma$ and $\mathrm{K}_{\mathrm{L}} \rightarrow \pi^{0} \pi^{0}$ branching ratios. This may be compared with the result of NA31: $\mathrm{BR}\left(\mathrm{K}_{\mathrm{L}} \rightarrow \mathrm{e}^{+} \mathrm{e}^{-} \gamma\right)=$ $(9.2 \pm 0.5 \pm 0.5) \times 10^{-6}[6]$ and the E845 measurement of $\left(9.1 \pm 0.4{ }_{-0.5}^{+0.6}\right) \times 10^{-6}[7]$. Both results would increase slightly if updated in the light of later values for intermediate branching ratios.

By the end of the projected running for $\varepsilon^{\prime} \varepsilon$ data in NA48, it is likely that the number of analyzed $\mathrm{K}_{\mathrm{L}} \rightarrow \mathrm{e}^{+} \mathrm{e}^{-} \gamma$ decays will have increased by an order of magnitude. Correspondingly there is some scope for improvement of systematic errors.

\section{Acknowledgements}

It is a pleasure to thank the technical staff of the participating laboratories, universities and affiliated computing centres for their efforts in the construction of the NA48 apparatus, in operation of the experiment, and in the processing of data. We are also grateful to H.B. Greenlee for making his code for radiative corrections available to us. 


\section{References}

[1] L.M. Sehgal, Phys. Rev. D7, 3303 (1973).

T. Miyazaki, Lett. Nuovo Cimento 3, 294 (1972).

[2] J.J. Sakurai, Phys. Rev. 156, 1508 (1967).

[3] M.A. Shifman et al., Nucl. Phys. B 120, 316 (1977).

[4] J.L. Goity and Longzhe Zang, Phys. Lett. B 398, 387 (1997).

[5] L. Bergström et al., Phys. Lett. B 131, 229 (1983).

[6] G.D. Barr et al., Phys. Lett. B 240, 283 (1990).

[7] K.E. Ohl et al., Phys. Rev. Lett. 65, 1407 (1990).

[8] M.B. Spencer et al., Phy. Rev. Lett. 74, 3323 (1995).

[9] V. Fanti et al., Z. Phys. C 76, 653 (1997).

[10] "The Beam and Detector for the Precision CP Violation Experiment NA48", to be submitted to Nucl. Inst. Meth.

[11] N.M. Kroll and W. Wada, Phys. Rev. 98, 1355 (1955).

[12] H. B. Greenlee, Phys. Rev. D 42, 3724 (1990). See also K.O. Mikaelian and J. Smith, Phys. Rev. D5, 1763 (1972) and D5, 2890 (1972), and L. Roberts and J. Smith, Phys. Rev. D33, 3457 (1986).

[13] Particle Data Group, Eur. Phys. J. C 3, 357 (1998).

[14] Particle Data Group, Eur. Phys. J. C 3, 460 (1998).

[15] Particle Data Group, Eur. Phys. J. C 3, 461 (1998). 